\title{
Cognitive Impairment and its Effect on Chronic Obstructive Pulmonary Disease: An Underestimated Phenomenon
}

\author{
Sachin L. Patel, Jagdish R. Varma', Jaishree D. Ganjiwale², Nimit V. Khara, Ravish M. Kshatriya, Rajiv P. Paliwal, Sateesh N. Patel \\ Departments of Respiratory Medicine and ${ }^{1}$ Psychiatry, Pramukhswami Medical College, ${ }^{2}$ Department of Community Medicine, Pramukhswami Medical College and \\ Central Research Services, HM Patel Center for Medical Care and Education, Karamsad, Gujarat, India
}

\section{Abstract}

Background: To assess the ability of the Rowland Universal Dementia Assessment Scale (RUDAS) to predict inhaler technique and determine the prevalence of cognitive impairment in chronic obstructive pulmonary disease (COPD). Patients and Methods: This cross-sectional study included 98 participants, and we recorded age, education, locality, occupation, COPD stage, comorbidities, duration of inhaler use, and smoking history. Inhaler technique was assessed using an internationally accepted schedule (nine steps). RUDAS, a multicultural cognitive assessment scale, was used to assess cognitive function. Results: The study included 92 males and 6 females with the mean age of 63.9 years. At a cutoff of 24 , RUDAS had $93.6 \%$ sensitivity and $88.2 \%$ specificity in screening incorrect inhaler technique. Those having a RUDAS score $<24$ were 272 times more likely to perform incorrect inhaler technique. On comparison of incorrect and correct inhaler technique groups on various parameters in univariate analysis, it was found that the groups were significantly different in terms of education, locality, occupation, COPD stage, comorbidities, and smoking history. However, in logistic regression, only RUDAS score $<24$ was found to be a predictor of incorrect inhaler technique. At the original cutoff of 23, RUDAS screened $42.9 \%(n=42)$ of the patients as having cognitive impairment. Conclusions: High prevalence of cognitive impairment among COPD patients and its untoward impact on inhaler technique is an important but underestimated clinical issue. RUDAS is an effective tool to predict incorrect inhaler technique and cognitive impairment in COPD.

Keywords: Chronic obstructive pulmonary disease, cognitive impairment, inhaler technique

\section{INTRODUCTION}

Chronic obstructive pulmonary disease (COPD) is a common respiratory condition, affecting $10 \%-15 \%$ of adults worldwide. ${ }^{[1]}$ Its prevalence is on the rise, while the incidence in old age continues to grow. ${ }^{[2]}$ It can lead to significant reduction in physical activity and psychological problems, all of which deteriorate the patient's health-related quality of life. ${ }^{[3]}$ COPD is a significant cause of mortality worldwide, amounting to $5 \%$ of deaths in the year $2015 .{ }^{[4]}$ COPD can also be associated with various comorbidities such as reduced cardiac function, poor nutrition, anemia, loss of muscle mass, osteoporosis, cognitive impairment, clinical depression, anxiety, and gastrointestinal reflux disorder. ${ }^{[4]}$

Trouble with memory, new learning, and decision-making in day-to-day life are symptoms of cognitive impairment. COPD has been found to be associated with increased risk of cognitive impairment. ${ }^{[5]}$ Cognitive impairment in COPD has been postulated due to a repeated exposure to hypoxia, leading to impaired functioning of enzymes required for neurotransmitter

\begin{tabular}{|l|l|}
\hline \multicolumn{2}{|c|}{ Access this article online } \\
\hline Quick Response Code: & Website: \\
\hline
\end{tabular}

synthesis, eventually leading to neural dysfunction. ${ }^{[6,7]}$ Recent research using functional magnetic resonance imaging has found that COPD individuals demonstrate loss of white matter as compared to controls. This may lead to disturbed gray matter activation and cognitive dysfunction. ${ }^{[8]}$ Executive function, memory, and attention are involved in COPD-related cognitive dysfunction. ${ }^{[9]}$ Impairments in executive functioning impair planning, goal setting, sequencing, execution, and monitoring. Previous research has found that cognitive impairment (drawing impairment) is a risk factor for mortality in severe COPD patients. ${ }^{[10]}$

Address for correspondence: Dr. Jagdish R. Varma Department of Psychiatry, Pramukhswami Medical College Karamsad - 388 325, Anand, Gujarat, India E-mail: jagdishrv@charutarhealth.org

This is an open access journal, and articles are distributed under the terms of the Creative Commons Attribution-NonCommercial-ShareAlike 4.0 License, which allows others to remix, tweak, and build upon the work non-commercially, as long as appropriate credit is given and the new creations are licensed under the identical terms.

For reprints contact: reprints@medknow.com

How to cite this article: Patel SL, Varma JR, Ganjiwale JD, Khara NV, Kshatriya RM, Paliwal RP, et al. Cognitive impairment and its effect on chronic obstructive pulmonary disease: An underestimated phenomenon. Indian J Respir Care 2020;9:82-7.

Received: $15-05-2019$

Accepted: 10-10-2019

Revised: 08-10-2019 Published: 08-01-2020 
Patel, et al.: Brief cognitive test to predict inhaler use

Proper inhaler technique has been found to be a predictor of efficacy of therapy. ${ }^{[1]}$ However, the ability of patients to properly handle the device may get less importance among other things. This can lead to unnecessary escalation of treatment and hospitalization. An expert panel on inhaler adherence has recommended that improved knowledge of errors may help treating doctors in early identification and corrective measures. ${ }^{[12]}$ Hence, there is a need to identify clinical methods that can predict patient's proficiency for inhaler technique. Previous research has found association between executive dysfunction and poor inhaler technique. ${ }^{[13]}$

There are limited studies from rest of the world, done to predictability to learn inhaler technique using cognitive tests, ${ }^{[14-16]}$ and none from India, which was the primary aim of the study. The secondary aim of the study was to assess the prevalence of cognitive impairment in COPD patients.

\section{Patients and Methods}

A cross-sectional study was conducted among COPD patients coming to the outpatient department and/or admitted under the care of department of respiratory medicine at the institute and meeting inclusion and exclusion criteria, over a period of 6 months (from March 2018 to September 2018). Inclusion criteria were (a) adult patient of any age, gender; diagnosed with COPD using the Global Initiative for Chronic Obstructive Lung Disease (GOLD) guidelines (2017); (b) who have been clinically stable in the last 4 weeks prior to the study; and (c) who were on regular inhaler therapy since at least previous 2 months. Patients with a previous diagnosis of mental illness or cognitive dysfunction were excluded from the study.

Ethical approval was obtained from the Institutional Ethics Committee. Written informed consent was obtained from the participants before enrolment. A convenience sample of 100 patients with COPD was included.

The study data were collected under five headings: (1) Demography, (2) COPD status, (3) Comorbidities, (4) Evaluation of inhaler technique, and (5) Cognitive status. Demographic data included age, gender, locality, education, occupation, and smoking status. COPD status included duration of illness, severity of illness (using pulmonary function test report and GOLD criteria), medications that the participants were using, duration of inhaler use, number of times participant has been trained to use inhaler on earlier occasions by physician and other health-care workers, and oxygen saturation on the day of evaluation - was assessed using portable $\mathrm{SpO}_{2}$ monitor. Comorbidities based on clinical history (history of hypertension, diabetes, congestive heart failure, or any other). Inhaler technique assessed using an internationally accepted inhaler use schedule was used to assess the current inhaler technique of the participant [Box 1]. ${ }^{[17]}$

Cognitive function was assessed based on performance on the items of the Rowland Universal Dementia Assessment Scale (RUDAS). ${ }^{[18]}$ RUDAS was chosen because it is

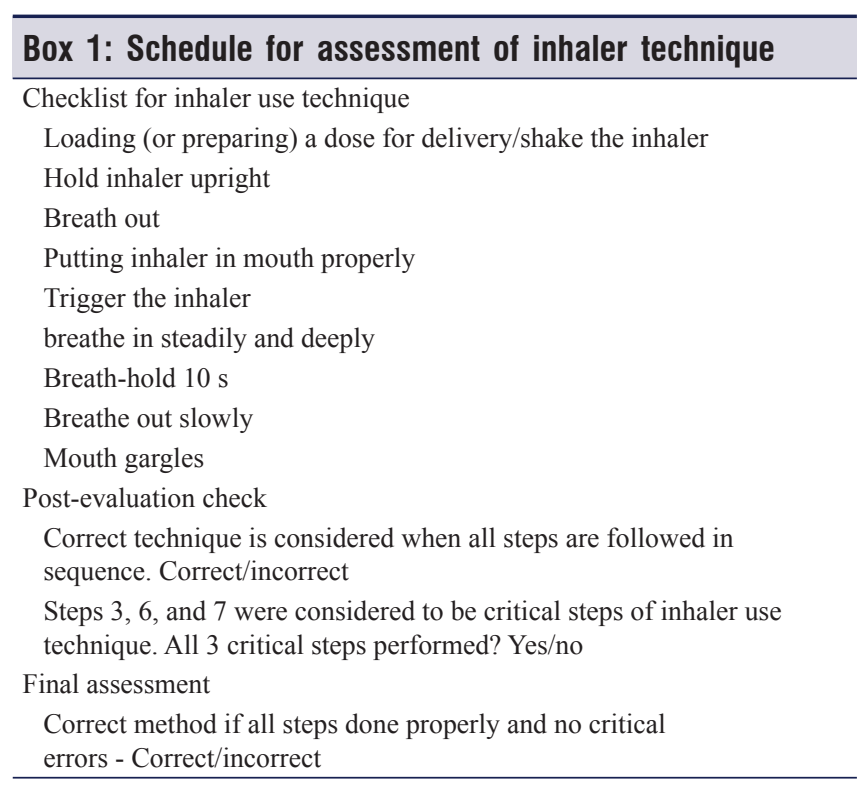

designed as a multicultural cognitive assessment scale which has set guidelines for use with a diverse set of population and guidelines for use in a different language than English. RUDAS is available in public domain and does not require permission to translate in another language. Gujarati version of RUDAS was developed using translation-back translation method. Face validity was assessed by agreement among the investigators and opinion of two psychiatrists, who were not part of the study, was taken. During pilot testing, it was found that three-dimensional wireframe used for assessment of Step 4, visuoconstructional drawing, was difficult for our patients. Hence, this was substituted with "Box in a Box" figure from the Hindi Mental Status Examination (HMSE).$^{[19]}$ This figure was used because the original scoring could be applied to this figure.

\section{Statistical analysis}

Data were entered in Microsoft Excel, and codes were assigned to raw data. Data were analyzed using STATA version 14, StataCorp, Texas, USA and JAMOVI 0.9.1.6, www.jamovi. org. Descriptive statistics were calculated for demographic data, COPD profile, comorbidities, and inhaler technique. Ability to use inhaler was considered incorrect if any of the steps were done incorrectly [Box 1]. Receiver operating characteristic (ROC) curve and cutoff score for ability of RUDAS score in predicting incorrect inhaler technique was calculated. Demographics, disease, medication, comorbidity, and smoking-related factors associated with incorrect inhaler technique were explored using cross-tabulation, and Chi-square test was used to assess significance of difference. The threshold for significant difference in this study was 0.05 . Variables were entered in the logistic regression model for incorrect inhaler technique: age, education category, rural/ urban, occupation, COPD stage, any comorbidity, duration of inhaler use, smoker type, and RUDAS category ( $<24$ and $\geq 24$, based on ROC analysis). Prevalence of cognitive impairment in COPD patients was calculated using the original RUDAS 
cutoff score of 23/30. Correlation of age with RUDAS score was calculated.

\section{RESULTS}

One-hundred patients were included in the study. Ninety-eight were included in the analysis (two patients, who were never trained by any health-care worker for inhaler use, were excluded). The study population included 92 males $(93.9 \%)$ and 6 females (6.1\%). Mean (standard deviation [SD]) age of the study population was 63.9 (9.3) years. Three-fourths $(n=74$, $75.5 \%)$ were from rural areas. Fifty-seven percent $(n=56)$ had 7 or more years of education. Majority of the population $(86.7 \%)$ represented semiskilled workers or laborers. Almost half $(n=50,51 \%)$ were chronic smokers [Table 1$]$.

More than 90\% $(n=90)$ have moderate-to-severe COPD, according to the GOLD criteria. Forty patients $(41.8 \%)$ had comorbid medical conditions. About $80 \%(n=78)$ were on both oral medications and inhalers [Table 2].

Ninety-nine percent $(n=97)$ were using dry powder inhalers. Majority of the patients $(98 \%)$ had been trained for inhaler use technique by respiratory physician, with a median of 5 explanations for the study population. More than three-fourth $(n=76,77.6 \%)$ of the patients had never been explained the inhaler procedure by any other health-care worker. Forty-eight percent $(n=47)$ demonstrated correct method of inhaler use. Fifty-two percent $(n=53)$ made errors on sequence of inhaler use and $48 \%(n=49)$ made critical step errors. On the nine steps of the inhaler technique, our study participants had a mean (SD) score of $7.8(1.25)$ correct steps $(n=98)$.

Figure 1 shows the ROC curve for ability of RUDAS score in predicting incorrect inhaler technique. The area under the curve (AUC) was 0.922 (95\% confidence interval [CI] [86.3, 98.2]). At a cutoff of $23.5 / 24$, RUDAS had $93.6 \%$ sensitivity

\begin{tabular}{llc}
\hline $\begin{array}{l}\text { Table 1: Demographic profile of the study } \\
\text { population }(\boldsymbol{n = 9 8 )}\end{array}$ & \\
\hline Variable & Characteristic & $\boldsymbol{n}(\%)$ \\
\hline Age & Mean (SD) & $63.9(9.3)$ \\
& Minimum-maximum & $45-90$ \\
Gender & Male & $92(93.9)$ \\
& Female & $6(6.1)$ \\
Locality & Rural & $74(75.5)$ \\
& Urban & $24(24.5)$ \\
Years of formal education & $0-6$ & $42(42.9)$ \\
& 7 or more & $56(57.1)$ \\
Occupation & Skilled & $5(5.1)$ \\
& Semi-skilled & $41(41.8)$ \\
& Laborer & $44(44.9)$ \\
& Homemaker & $4(4.1)$ \\
& Unemployed & $4(4.1)$ \\
Smoker type & Chronic smoker & $50(51)$ \\
& Ex-smoker & $26(26.5)$ \\
& Never smoker & $22(22.4)$ \\
\hline
\end{tabular}

SD: Standard deviation and $88.2 \%$ specificity in screening incorrect inhaler technique. At this cutoff, RUDAS has a positive likelihood ratio $(\mathrm{LR})=7.93$ and negative $\mathrm{LR}=0.072$ for screening incorrect inhaler technique.

ROC curve for ability of RUDAS visuoconstructional task (draw box in bow) in predicting incorrect inhaler technique was also calculated (figure not shown). The score for this question was recoded into $0=$ mistake in any of the tasks and $1=$ able to do all tasks in question. The AUC was 0.615 , and it had a $93.6 \%$ sensitivity and $29.4 \%$ specificity in screening incorrect inhaler technique.

On comparison of the incorrect and correct inhaler technique groups on various parameters in univariate analysis, it was found that the groups did not differ in terms of age or duration of inhaler use. However, they were significantly different in terms of education, locality, occupation, COPD stage, comorbidities, and history of smoking [Table 3].

The following variables were entered in the logistic regression model for incorrect inhaler technique: age, education category, rural/urban, occupation, COPD stage, any comorbidity, duration of inhaler use, smoker type, and RUDAS category ( $<24$ and $\geq 24$ ). Of these, only RUDAS score $<24$ was found to be a predictor of incorrect inhaler technique [Table 4].

Baseline model could predict incorrect inhaler technique, 52\% of the time (same as tossing a coin). Once the variables were entered in the model, omnibus test of model coefficients found significant improvement over the baseline model $(P<0.001)$. Nagelkerke $R^{2}(0.80)$ suggests that the entered model could explain $80 \%$ variation in the outcome. The model was a good fit for the data as the Hosmer and Lemeshow test, $P=0.107$ ( $>0.05)$. The model could correctly predict incorrect inhaler technique $94 \%$ times. Those having a RUDAS score $<24$ are 272 times more likely than those having a normal score to perform incorrect inhaler technique.

At the original cutoff of $23 / 30$, RUDAS was screened $42.9 \%(n=42)$ of the patients as having cognitive

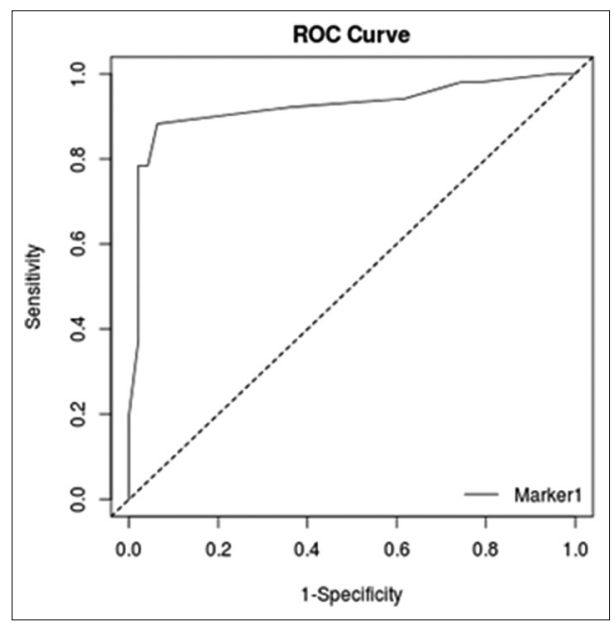

Figure 1: Receiver operating characteristic curve for Rowland Universal Dementia Assessment Scale score as a predictor of inhaler technique 
Patel, et al.: Brief cognitive test to predict inhaler use

dysfunction and $57.1 \%(n=56)$ as not having cognitive dysfunction. Correlation coefficient of RUDAS score with education was $0.639(P<0.001)$. Mean $(\mathrm{SD})$ score of participants with RUDAS score $<23$ on the steps of inhaler technique (6.69 [0.75], $n=42$ ) was significantly lower than mean (SD) of participants with RUDAS score $>23$ was $(8.63[0.84], n=56, P \leq 0.0001)$.

\begin{tabular}{llc}
\hline $\begin{array}{l}\text { Table 2: Chronic obstructive pulmonary disease profile of } \\
\text { the study population }(\boldsymbol{n}=\mathbf{9 8})\end{array}$ & \\
\hline Variable & Characteristic & $\boldsymbol{n}(\%)$ \\
\hline COPD stage & Mild & $8(8.2)$ \\
& Moderate & $67(68.4)$ \\
& Severe & $23(23.5)$ \\
Comorbidities* & Yes & $40(40.8)$ \\
& No & $58(59.2)$ \\
Current medications & Only oral & $1(1)^{* * *}$ \\
& Inhaled & $19(19.4)$ \\
& Both oral and inhaled & $78(79.6)$ \\
Type of medications & SABA & $97(99)$ \\
& SAMA & $40(40.8)$ \\
& LABA & $92(93.9)$ \\
& LAMA & $9(9.2)$ \\
& ICS & $92(93.9)$ \\
& Oral bronchodilator & $78(79.6)$ \\
\hline
\end{tabular}

*Hypertension 38 (38.8), diabetes mellitus 15 (15.3), Other 1 (1), **With past history of inhaler use. COPD: Chronic obstructive pulmonary disease, SABA: Short Acting Beta-2 Agonist, SAMA: Short Acting Muscarinic Antagonist, LABA: Long Acting Beta-2 Agonist, LAMA: Long Acting Muscarinic Antagonist, ICS: Inhaled Corticosteroid

\section{DISCUSSION}

COPD is considered a respiratory disease, but it can cause disabling extrapulmonary effects including cognitive impairment. Cognitive impairment can hamper the patient's proficiency to handle the inhaler device. We found that at a cutoff of 24/30 RUDAS had $93.6 \%$ sensitivity and $88.2 \%$ specificity in predicting incorrect inhaler technique. A study by Gray et al. to predict inhaler technique by using Mini-Mental Status Examination (MMSE) found that a score $<24$ had $54.8 \%$ sensitivity and $80 \%$ specificity (odds ratio, 3.66; $95 \%$ CI, 1.07-12.4) in predicting incorrect inhaler technique. ${ }^{[14]}$ Allen et al. used four cognitive tests to predict proficiency of metered-dose inhaler use, MMSE was one of them, which had a sensitivity of $57 \%$ and specificity of $76 \%{ }^{[15]}$

A pilot study done to compare MMSE and HMSE reported that both tools are variable and weak to classify cognitive impairment. ${ }^{[20]}$ These preliminary results suggest that in our population where MMSE has limitations of use due to language and education bias, RUDAS has better sensitivity and specificity in predicting the ability to use inhaler. RUDAS is simpler, less language, and educationally biased tool that can be used for screening patients who may have difficulties in learning inhaler use.

In our study, RUDAS visuoconstructional task (draw box in a box) had $93 \%$ sensitivity and $29 \%$ specificity in predicting incorrect inhaler technique. Quite similarly, Board and Allen had done a study using overlapping pentagons and reported that those who were doing poorly in the drawing test were unable to

\begin{tabular}{|c|c|c|c|c|}
\hline Variable & Characteristic & Incorrect inhaler technique $(n=51)$ & Correct inhaler technique $(n=47)$ & $P$ \\
\hline Age (years) & Mean (SD) & $62.8(8.1)$ & $65.0(10.5)$ & 0.25 \\
\hline \multirow[t]{2}{*}{ Education (years) } & $0-6$ & $30(51.8)$ & $12(25.5)$ & 0.001 \\
\hline & 7 or more & $21(41.2)$ & $35(74.5)$ & \\
\hline \multirow[t]{2}{*}{ Locality } & Rural & $45(88.2)$ & $29(61.7)$ & 0.002 \\
\hline & Urban & $6(11.8)$ & $18(38.3)$ & \\
\hline \multirow[t]{5}{*}{ Occupation } & Skilled & $1(2.0)$ & $4(8.5)$ & 0.007 \\
\hline & Semi-skilled & $14(27.4)$ & $27(57.4)$ & \\
\hline & Laborer & $31(60.8)$ & $13(27.7)$ & \\
\hline & Homemaker & $3(5.9)$ & $1(2.1)$ & \\
\hline & Unemployed & $2(3.9)$ & $2(4.3)$ & \\
\hline \multirow[t]{3}{*}{ COPD stage } & Mild & $6(11.8)$ & $2(4.2)$ & 0.012 \\
\hline & Moderate & $28(54.9)$ & $39(83.0)$ & \\
\hline & Severe & $17(33.3)$ & $6(12.8)$ & \\
\hline \multirow[t]{2}{*}{ Any comorbidity } & Yes & $22(43.1)$ & $36(76.6)$ & 0.001 \\
\hline & No & $29(56.9)$ & $11(23.4)$ & \\
\hline Duration of inhaler use (years) & Mean (SD) & $2.39(1.28)$ & $2.87(1.40)$ & 0.07 \\
\hline \multirow[t]{3}{*}{ Smoker type } & Chronic smoker & $36(70.6)$ & $14(29.8)$ & $<0.001$ \\
\hline & Ex-smoker & $13(25.5)$ & $13(27.7)$ & \\
\hline & Never smoker & $2(3.9)$ & $20(42.5)$ & \\
\hline \multirow[t]{2}{*}{ RUDAS score } & $<24$ & $45(88.2)$ & $3(6.4)$ & $<0.001$ \\
\hline & $24-30$ & $6(11.8)$ & $44(93.6)$ & \\
\hline
\end{tabular}

SD: Standard deviation, COPD: Chronic obstructive pulmonary disease, RUDAS: Rowland Universal Dementia Assessment Scale 
Patel, et al.: Brief cognitive test to predict inhaler use

\begin{tabular}{|c|c|c|c|c|}
\hline Variable & $B$ & Significance & Adjusted OR & $95 \% \mathrm{Cl}$ \\
\hline Education category & 0.363 & 0.762 & 1.437 & $0.138-14.962$ \\
\hline Rural/urban & -0.679 & 0.567 & 0.507 & $0.050-5.196$ \\
\hline Occupation category & & 0.785 & & \\
\hline Unemployed & Reference & & & \\
\hline Skilled & 0.041 & 0.983 & 1.042 & $0.022-48.423$ \\
\hline Semi-skilled & -1.936 & 0.268 & 0.144 & $0.005-4.448$ \\
\hline Laborer & -1.607 & 0.402 & 0.200 & $0.005-8.590$ \\
\hline Homemaker & -1.447 & 0.626 & 0.235 & $0.001-79.057$ \\
\hline COPD stage & & 0.655 & & \\
\hline Mild COPD & Reference & & & \\
\hline Moderate COPD & -0.197 & 0.899 & 0.821 & $0.038-17.565$ \\
\hline Severe COPD & 0.863 & 0.639 & 2.371 & $0.064-87.265$ \\
\hline \multicolumn{5}{|l|}{ Any comorbidity } \\
\hline Yes & 0.126 & 0.909 & 1.134 & $0.131-9.831$ \\
\hline No & Reference & & & \\
\hline Smoker type & & 0.148 & & \\
\hline Chronic smoker & 2.705 & 0.134 & 14.956 & $0.436-513.292$ \\
\hline Ex-smoker & 4.008 & 0.051 & 55.012 & $0.986-3068.179$ \\
\hline RUDAS score $<24$ & 5.607 & $<0.001$ & 272.442 & $18.396-4034.912$ \\
\hline
\end{tabular}

learn inhaler use (93\% specificity). ${ }^{[16]}$ While Allen et al. found a $75 \%$ sensitivity and $79 \%$ specificity in predicting incorrect inhaler technique using intersecting pentagons. ${ }^{[15]}$ They also used a clock-drawing task (CLOX) as a measure of executive function in predicting incorrect inhaler technique. They found that CLOX1 score $<10$ had $83 \%$ sensitivity and $57 \%$ specificity, whereas CLOX2 score $<12$ had $58 \%$ sensitivity and $64 \%$ specificity in predicting incorrect inhaler technique. Hence, in settings when the clinician is pressed for time, the RUDAS drawing task can yield good sensitivity in predicting ability to use inhaler. Furthermore, it can be given to the patient to perform while they are in the waiting area.

In our study, representing mainly participants with moderate-to-severe COPD, $42 \%$ screened positive for cognitive impairment at the original cutoff of 23/30 for RUDAS. In an Indian study done by Gupta et al., to assess cognitive impairment in nonhypoxemic COPD patients using MMSE and P300 test, they found that 10 out of their $40(25 \%)$ patients demonstrated prolonged P300 latency and 27 out of $40(67.5 \%)$ patients had MMSE scores lower than $99^{\text {th }}$ percentile healthy volunteers. ${ }^{[21]}$

High prevalence of cognitive impairment in our study population raises awareness about the need for addressing this problem in our clinical settings. This is particularly necessary as cognitive impairment can lead to trouble in remembering, learning new things, concentrating, or making decision. This can make handling money and medications (part of the instrumental activities of daily living [IADL]) difficult. This is corroborated by research which has shown that increase in cognitive impairment over the course of illness is associated with reduction in IADL scores. ${ }^{[2]}$
Andrianopoulos et al. have recommended that health-care professionals must be mindful of cognitive impairment in their COPD patients and when present should be a trigger for better rehabilitation. ${ }^{[23]}$ Findings of above and our study suggest that regular screening of cognition must be done in COPD patients.

\section{Strengths of the study}

Investigators were mindful on developing inclusion criteria such that finally, $98 \%$ participants had been trained for inhaler use technique by respiratory physician, with a median of five explanations per participant. Despite this level of training, $48 \%$ of the patients had incorrect inhaler technique on evaluation. No previous work on these lines has been done in Indian settings.

Cross-sectional study, small sample size, male bias, and small representation of patients having mild illness are some of the limitations of this study. For screening of cognitive dysfunction RUDAS demonstrated education effects. Further research is required with education neutral or less educationally biased locally developed tools in a larger sample size on drug-naïve patients, who are serially followed up to evaluate natural course of ability to use inhaler.

\section{Conclusions}

High prevalence of cognitive impairment among COPD patients and its untoward impact on inhaler technique is an important but underestimated clinical issue. RUDAS is an effective tool to predict incorrect inhaler technique and cognitive impairment in COPD.

\section{Acknowledgments}

The authors acknowledge the contribution of Dr. Anusha Prabhakaran, Professor and Dr. Aamir Pathan, Senior Resident, 
from the Department of Psychiatry who helped in judging face validity of Gujarati translation of RUDAS.

\section{Financial support and sponsorship}

Self-funded.

\section{Conflicts of interest}

There are no conflicts of interest.

\section{RefERENCES}

1. Ries AL, Carlin BW, Carrieri-Kohlman V, Casaburi R, Celli BR, Emery CF, et al. Pulmonary rehabilitation: Joint ACCP/AACVPR evidence-based guidelines. Chest 1997;112:1363-96.

2. Vestbo J, Hurd SS, Agustí AG, Jones PW, Vogelmeier C, Anzueto A, et al. Global strategy for the diagnosis, management, and prevention of chronic obstructive pulmonary disease: GOLD executive summary. Am J Respir Crit Care Med 2013;187:347-65.

3. Lopez AD, Shibuya K, Rao C, Mathers CD, Hansell AL, Held LS, et al. Chronic obstructive pulmonary disease: Current burden and future projections. Eur Respir J 2006;27:397-412.

4. Divo M, Cote C, de Torres JP, Casanova C, Marin JM, Pinto-Plata V, et al. Comorbidities and risk of mortality in patients with chronic obstructive pulmonary disease. Am J Respir Crit Care Med 2012;186:155-61.

5. Samareh Fekri M, Hashemi-Bajgani SM, Naghibzadeh-Tahami A, Arabnejad F. Cognitive impairment among patients with chronic obstructive pulmonary disease compared to normal individuals. Tanaffos 2017;16:34-9.

6. Heaton RK, Grant I, McSweeny AJ, Adams KM, Petty TL. Psychologic effects of continuous and nocturnal oxygen therapy in hypoxemic chronic obstructive pulmonary disease. Arch Intern Med 1983;143:1941-7.

7. Lee JM, Grabb MC, Zipfel GJ, Choi DW. Brain tissue responses to ischemia. J Clin Invest 2000;106:723-31.

8. Dodd JW. Lung disease as a determinant of cognitive decline and dementia. Alzheimers Res Ther 2015;7:32.

9. Incalzi RA, Gemma A, Marra C, Capparella O, Fuso L, Carbonin P. Verbal memory impairment in COPD: Its mechanisms and clinical relevance. Chest 1997;112:1506-13.

10. Antonelli-Incalzi R, Corsonello A, Pedone C, Trojano L, Acanfora D, Spada A, et al. Drawing impairment predicts mortality in severe COPD. Chest 2006;130:1687-94.

11. Bonini M, Usmani OS. The importance of inhaler devices in the treatment of COPD. COPD Res Pract 2015;1:9.

12. Braido F, Chrystyn H, Baiardini I, Bosnic-Anticevich S, van der Molen T, Dandurand RJ, et al. "Trying, but failing" - The role of inhaler technique and mode of delivery in respiratory medication adherence. J Allergy Clin Immunol Pract 2016;4:823-32.

13. Kirkil G, Tug T, Ozel E, Bulut S, Tekatas A, Muz MH. The evaluation of cognitive functions with $\mathrm{P} 300$ test for chronic obstructive pulmonary disease patients in attack and stable period. Clin Neurol Neurosurg 2007;109:553-60.

14. Gray SL, Williams DM, Pulliam CC, Sirgo MA, Bishop AL, Donohue JF. Characteristics predicting incorrect metered-dose inhaler technique in older subjects. Arch Intern Med 1996;156:984-8.

15. Allen SC, Warwick-Sanders M, Baxter M. A comparison of four tests of cognition as predictors of inability to learn to use a metered dose inhaler in old age. Int J Clin Pract 2009;63:1150-3.

16. Board M, Allen SC. A simple drawing test to identify patients who are unlikely to be able to learn to use an inhaler. Int J Clin Pract 2006;60:510-3.

17. Restrepo RD, Alvarez MT, Wittnebel LD, Sorenson H, Wettstein R, Vines DL, et al. Medication adherence issues in patients treated for COPD. Int J Chron Obstruct Pulmon Dis 2008;3:371-84.

18. Storey JE, Rowland JT, Basic D, Conforti DA, Dickson HG. The Rowland universal dementia assessment scale (RUDAS): A multicultural cognitive assessment scale. Int Psychogeriatr 2004;16:13-31.

19. Ganguli M, Ratcliff G, Chandra V, Sharma S, Gilby J, Pandav R. A Hindi version of the MMSE: The development of a cognitive screening instrument for a largely illiterate rural elderly population in India. Int J Geriatr Psychiatry 1995;10:367-77.

20. Tiwari SC, Tripathi RK, Kumar A. Applicability of the mini-mental state examination (MMSE) and the Hindi mental state examination (HMSE) to the urban elderly in India: A pilot study. Int Psychogeriatr 2009;21:123-8.

21. Gupta PP, Sood S, Atreja A, Agarwal D. A comparison of cognitive functions in non-hypoxemic chronic obstructive pulmonary disease (COPD) patients and age-matched healthy volunteers using mini-mental state examination questionnaire and event-related potential, P300 analysis. Lung India 2013;30:5-11.

22. Sloan FA, Wang J. Disparities among older adults in measures of cognitive function by race or ethnicity. J Gerontol B Psychol Sci Soc Sci 2005;60:P242-50.

23. Andrianopoulos V, Gloeckl R, Vogiatzis I, Kenn K. Cognitive impairment in COPD: Should cognitive evaluation be part of respiratory assessment? Breathe (Sheff) 2017;13:e1-9. 